\title{
Cognition in adult migraineurs: An electrophysiological study
}

\author{
Pooja Ojha ${ }^{1, *}$, Varun Malhotra ${ }^{2}$, Nitin Pandey ${ }^{3}$, Zeashan Zaidi ${ }^{4}$ \\ ${ }^{\mathbf{1 , 2 , 4}}$ Assistant Professor, ${ }^{\mathbf{3}}$ Associate Professor, ${ }^{1,3}$ Dept. of Physiology, ${ }^{2}$ Dept. of Neurology, ${ }^{4}$ Dept. of Community \\ Medicine, Era's Lucknow Medical College, Sarfarazganj, Lucknow, Uttar Pradesh, India
}

*Corresponding Author:

Email: drpojha@gmail.com

\begin{abstract}
Introduction: Researches have shown that migraine causes clinically dormant brain lesions which can lead to gradual deterioration of cognitive functions in migraineurs.

Objective: The purpose of current study was to elucidate cognitive processing in migraine patients, using auditory event related P300 potential, as results of previous work in this field has not been very conclusive.

Materials and Methods: 25 Migraine patients (8 males, 17 females) from neurology OPD of the associated hospital and 25 healthy age and gender matched controls were recruited for the study. Study protocol included a thorough history taking followed by clinical neurological examination. Migraine disability assessment (MIDAS) score was obtained from MIDAS questionnaire. Participants were subjected to auditory P300 potential recording using oddball paradigm. Responses to target stimuli were averaged and Latencies (ms) of the P300 peak and amplitudes $(\mu \mathrm{v})$ of P300 were recorded.

Results: P300 target stimulus latency in patients with Migraine was $349.48 \pm 38.50 \mathrm{~ms}$ (Mean \pm SD) and in healthy controls, it was $307.09 \pm 37.18$ (Mean \pm SD). This was statistically significant $(\mathrm{P}<0.001)$. No significant changes were observed in P300 amplitude between cases and control. Neither P300 target stimulus latency nor P300 target stimulus amplitude, showed any significant correlation with MIDAS score.

Conclusion: Decline in cognitive functions in patients with migraine does occur as seen with prolonged P300 latency when compared to healthy controls. In time to come, P300 may become an important tool for clinicians to assess certain aspects of cognition.
\end{abstract}

Keywords: Cognition, P300, Event related potential, MIDAS score, Migraine.

Received: $26^{\text {th }}$ October, 2017

\section{Introduction}

Headache disorders together are the third highest cause of years lived with disability $(\mathrm{YLDs})^{1}$ and Migraine, a type of primary headache disorder, is at number six among the causes of YLD, by the Global Burden of Disease 2013. ${ }^{2}$ Migraine involves the neural and cerebrovascular system, and few patients present with transient neurological symptoms, known as migraine aura. Some previous researches have shown that migraine is a potent risk factor for stroke. It can lead to occurrence of clinically dormant brain lesions. ${ }^{3-5}$ Presence of such structural lesions of brain, in patients of migraine predisposes them to gradual deterioration of cognitive functions. ${ }^{6}$

The mechanism of cognitive processing in the brain can be easily assessed with the P300 potential.P300 is an endogenous Late Auditory Evoked Potentials as it is more dependent on internal cognitive processes.P300 reflects cortical activity and depends upon some abilities, such as attention, discrimination and memory. ${ }^{7}$ Changes in P300 amplitude show the degree to which incoming information is processed and fed to the working memory. On the other hand, P300 latency is an estimate of stimulus classification speed, and increases as the cognitive capability decreases. ${ }^{8-10}$ Thus it can be understood that recording P300 evoked potential in patients of Migraine might reflect their cognitive abilities and extent of disease progression. Results of previous studies assessing cognitive dysfunction in Migraine patients are conflicting. Also there is scarcity of data which studies the correlation between P300 potential parameters and MIDAS score. The MIDAS (migraine disability assessment) questionnaire is used to assess the actual impact of headaches in last three months, on patient's life. ${ }^{11}$ There are studies which have tested the reliability of MIDAS score in patients with headache and it came out to be highly reliable. ${ }^{12}$ Therefore the current study aims to elucidate the cognitive processing in migraine patients, using auditoryP300 potential and it's correlation with MIDAS score.

\section{Materials and Methods}

Participants: Migraine patients were recruited from neurology OPD of the associated hospital. All Male and female patients between age group 20-45 years who fulfilled the diagnostic criteria 
of migraine according to the International Classification of Headache Disorder, third edition $^{13}$ with normal hearing threshold, were considered for the study. Study protocol included a thorough history taking followed by clinical neurological examination. History included socio-demographic details and particulars of Migraine history, such as duration of disease in years, presence or absence of aura, severity and impact of headache assessed through MIDAS questionnaire. Questions were explained to patients for their full cooperation. Patients with other neurological and psychiatric disease known to cause cognitive dysfunction and headache were excluded from the study. Those with impaired auditory function were also excluded from the study. Medication for Migraine was continued during the study. Control group comprised of randomly selected healthy volunteers with normal hearing and without any personal or family history of headache.

A Written, informed consent was taken from all participants. This study was approved by the Research Ethics committee of the institute. P300-auditory Event related potential was recorded in migraine patients irrespective of the headache period and compared with that of control group.

Recording of p300 evoked potential: P300 was recorded using Neurostim Medicaid polyrite system, in a sound attenuated room with subject lying comfortably on couch. Surface electrodes were attached to vertex $(\mathrm{Cz}$, active electrode) and mastoids (reference electrodes M1 and M2, left and right mastoid respectively) and on the forehead at Fpz (ground) with conductive paste and micropore tape, according to the International $10-20$ system. $^{14}$ The P300 component was elicited with a simple discrimination task known as the 'oddball' paradigm, in which two auditory stimuli (frequent \& rare) are presented. ${ }^{15}$ Pure tone auditory stimuli, with an intensity of $75 \mathrm{~dB}$ Sound Pressure Level (SPL) were presented in a random series to both ears through a headphone with frequent $(80 \%)$ tones of $1000 \mathrm{~Hz}$ and rare stimuli $(20 \%)$ of $2000 \mathrm{~Hz}$. The procedure was explained to the subjects to achieve full cooperation and they were familiarized with two types of auditory stimuli. They were instructed to close their eyes and make minimal eye movement. During recordings, subjects were instructed to mentally count rare stimuli target tones and report at the end. The equipment gives the number of target stimuli delivered. Only those sessions in which the participants achieved 90 percent accuracy in counting target stimuli were included. Finally, all responses to target stimuli were averaged and cursor was placed on screen to mark P300 peak. Latency (ms) of the P300 peak and amplitudes ( $\mu \mathrm{v})$ of P300 were recorded.

\section{Statistical analysis}

Data was analyzed using Statistical Package for Social Sciences, version 16.0. Independent samples't'-test and Pearson correlation was used to compare and correlate the data. ' $\mathrm{P}$ ' value < 0.05 was considered as significant.

\section{Result}

Study group comprised of a total of 25 cases of Migraine (8 males \& 17 females), Age mean $31.20 \pm 6.43$ y and 25 age and gender-matched controls, (8 males \& 17 females), Age mean $30 \pm$ 6.12 y. Details of migraine history has been presented in Table 1. [Table 1]

Table 1: Overview of migraine history in patients

\begin{tabular}{|l|}
\hline Migraine patients $\mathrm{N}=25$ \\
\hline Age of onset of headache in years (mean $\pm \mathrm{SD}) 21.32 \pm 5.963$ \\
\hline Duration of migraine history in years $($ mean \pm SD) $9.88 \pm 3.64$ \\
\hline Number of migraineurs $(\%)$ \\
Presence of aura $4(16 \%)$ \\
\hline Severity of headache: \\
MIDAS score (mean \pm SD) $15.28 \pm 3.82$ \\
\hline
\end{tabular}

P300 target stimulus latency at $\mathrm{Cz}$ electrode position in migraineurs was prolonged when compared to control group. No statistically significant difference was observed between P300 amplitude in migraineurs and controls [Table 2]

Table 2: P300 potentials of migraineurs and controls

migraineurs ( $\mathrm{n}=25)$ Controls $(\mathrm{n}=25) \mathrm{t}$-value $\mathrm{P}$ value
Mean (SD) Mean (SD)
P300 latency (ms) 349.48 (38.50) 307.09 (37.18) $-3.959 \mathrm{P}<$

Indian Journal of Clinical Anatomy and Physiology, January-March, 2018;5(1);77-80 
N2P3 amplitude $(\mu \mathrm{V}) 13.95(2.31) 14.12$ (3.62) $.204 \mathrm{P}=.839$

Correlation between MIDAS scores and P300 amplitude was weak negative and statistically not significant $(\mathrm{r}=-0.308 ; \mathrm{p}=0.134)$. Correlation between MIDAS scores and P300 latency was weak positive but statistically not significant $(\mathrm{r}=.162 ; \mathrm{p}=.438)$. [Table 3].

Table 3: Pearson correlation coefficient between P300 parameters and MIDAS score

\begin{tabular}{|l|}
\hline P300 latency $\mathrm{P} 300$ amplitude \\
\hline MIDAS score $\mathrm{r}=.162 ; \mathrm{P}=.438 \mathrm{r}=-.308 ; \mathrm{P}=.134$ \\
\hline
\end{tabular}

Presence of aura did not show a statistically significant association with either P300 latency or P300 amplitude ( $>>0.05)$.

\section{Discussion}

This study was designed to assess cognition in adult migraineurs using Late Auditory Evoked Potential P300. It is known that P300 is a neural signature of attention or the amount of working memory required for appropriately responding to environmental stimuli. Two important P300 parameters which are considered an objective index of cognitive processing are P300 latency and P300 amplitude. ${ }^{16}$ In our study, we used auditory oddball paradigm, to compare P300 target stimulus potentials between patients with migraine and control subjects. Previous researches have shown a variety of outcomes. Some have demonstrated prolongation of P300 latencies and an increase in P300 amplitude as well in migraineurs during headache attacks. ${ }^{17}$ Others found a reduction of P300 amplitude but no significant changes in P300 latency in migraineurs when compared to controls. ${ }^{18,19}$ Still some have shown that migraine patients have reduced P300 amplitude and longer P300 latency ${ }^{20,21}$ and finally in some studies prolongation in P300 latency was observed with no significant change in P300 amplitude in migraineurs when compared to controls. ${ }^{22,23}$ Our findings are consistent with these studies; the auditory ERP P300 latency was significantly prolonged in migraineurs at $\mathrm{Cz}$ electrode position as compared to the control group.

Cognition abnormalities in migraineurs can be explained by the fact that migraine is a chronic neurovascular disorder. The pathological processes in neural tissues hamper a smooth interaction between cortical and subcortical neural circuits, leading to delay in conduction and disturbances in cognitive processing. Also, as suggested by some authors, some cognitive functions can be modulated by head pain experience and probably frequent pain may produce a permanent abnormality of brain and cognition disturbances. ${ }^{24}$
Headache-related disabilities affect different aspects of daily life, this includes employment, household work, and non-work activities and this is assessed by a brief, self-administered MIDAS questionnaire. Studies show that MIDAS scores correlate well with physician judgments regarding patients' pain, disability, and need for medical care. Such findings strengthen the utility of the MIDAS questionnaire in clinical practice. ${ }^{25}$ But we did not find any significant correlation between the scores obtained through this questionnaire and P300 latency and amplitude. Probably because MIDAS does not assess the full spectrum of headache, and covers one third of the range between moderate and severe intensity.

This study has few limitations. Greater sample size to examine more number of patients and see the effect of gender on event related potential P300. Elimination of drug effect or interruption of therapy before recording would have yielded better information about ERP.

\section{Conclusion}

Decline in cognitive functions in patients with migraine does occur as seen with longer P300 latency in migraineurs when compared to healthy controls. In time to come, P300 may become an important tool for clinicians to assess certain aspects of cognition.

\section{Reference}

1. Steiner TJ, Birbeck GL, Jensen RH, Katsarava Z, Stovner LJ, Martelletti P (2015) Headache disorders are third cause of disability worldwide. J Headache Pain 16:58

2. Vos T, Barber RM, Bell B, Bertozzi-Villa A, Biryukov S, Bolliger I, Charlson F, Davis A, Degenhardt L, Dicker D, Duan L, Erskine H, Feigin VL, Ferrari AJ, Fitzmaurice C, Fleming T, Graetz N, Guinovart C, Haagsma J, Hansen GM, Hanson SW, Heuton KR, Higashi H, Kassebaum N, Kyu H, Laurie E, Liang X, Lofgren K, Lozano 
R, MacIntyre MF, Moradi-Lakeh M, Naghavi M, Nguyen G, Odell S, Ortblad K et al (2015) Global, regional, and national incidence, prevalence, and years lived with disability for 301 acute and chronic diseases and injuries in 188 countries, 1990-2013: a systematic analysis for the Global Burden of Disease Study 2013. Lancet 386:743-800

3. Kurth T, Kase CS, Schurks M, Tzourio C, Buring JE. Migraine and risk of haemorrhagic stroke in women: prospective cohortstudy. BMJ. 2010;341: c3659. doi: 10.1136/bmj.c3659.

4. Kurth T, Mohamed S, Maillard P, Zhu YC, Chabriat H, MazoyerB, et al. Headache, migraine, and structural brain lesionsand function: population based Epidemiology of Vascular Ageing-MRI study. BMJ. 2011;342:c7357. doi: 10.1136/bmj.c7357.

5. Kruit MC, van Buchem MA, Launer LJ, Launer LJ, TerwindtGM, Ferrari MD. Migraine is associated with an increased riskof deep white matter lesions, subclinical posterior circulationinfarcts and brain iron accumulation: the population-based MRI CAMERA study. Cephalalgia. 2010;30(2):129-36.

6. Kelman L. The postdrome of the acute migraine attack. Cephalalgia. 2006;26(2):214-20

7. Karniski W, Blair RC. Topographical and temporal stability of the P300.

Electroencephalogr Clin Neurophysiol. 1989;72(5):373-83.

8. Polich J. P300 clinical utility and control of variability. J Clin Neurophysiol 1998;15:14-33.

9. Polich J, Herbst KL. P300 as a clinical assay: rationale, evaluation andfindings. Int $\mathrm{J}$ Psychophysiol 2000;38:3-19.

10. Dochin E, Coles MGN. Is the P300 component a manifestation of context updating? Behav Brain Sci 1988;11:357-74.

11. Stewart WF, Lipton RB, Dowson A. J., and Sawyer J., "Development and testing of the Migraine Disability Assessment (MIDAS) Questionnaire to assess headache-related disability," Neurology, vol. 56, no. 6, supplement 1, pp. S20-S28,2001

12. Stewart WF, Lipton RB, Kolodner K, Liberman J, Sawyer J. Reliability of the migraine disability assessment score in a population-based sample of headache sufferers; Cephalalgia. 1999

Mar;19(2):107-14 ;discussion 74.

13. Headache Classification Committee of the International Headache Society(IHS) (2013) The International Classification of Headache Disorders, 3rd edition (beta version). Cephalalgia 33:629-808

14. Jasper HH. Appendix to report to committee on clinical examination in EEG: the ten-twenty electrode system of the international federation. Electroencephalogr Clin Neurophysiol. 1958;10:371-5

15. Polich J: P300 in clinical applications. In Electroencephalography: Basic Principles, Clinical Applications and Related fields, 4th ed. Edited by Niedermeyer E, Lopes Da Silva F. Urban and Schwarzenberg: Baltimore and Munich;1999:1073-91
16. Polich J. Updating P300: an integrative theory of P3a and P3b. Clin Neurophysiol. 2007;118(10): 2128-48.

17. Mazzotta G, Alberti A, Santucci A, Gallai V. The event-related potential P300 during headache-free period and spontaneous attack in adult headache sufferers. Headache. 1995;35(4):210-5.

18. Chen W, Shen X, Liu X, Luo B, Liu Y, Yu R, et al. Passive paradigm single-tone elicited ERPs in tension-type headaches and migraine. Cephalalgia. 2007;27(2):139-44.

19. Wang R, Dong Z, Chen X, Zhang M, Yang F, Zhang $X$, et al. Gender differences of cognitive function in migraine patients: evidence from event-related potentials using the oddball paradigm. J Headache Pain. 2014;15(1):6.

20. Drake ME, Pakalnis A, Padamadan H. Longlatency auditory event related potentials in migraine. Headache. 1989;29(4):239-41.

21. Rongfei Wang et al. "Gender differences of cognitive function in migraine patients: evidence from event-related potentials using the oddball paradigm". The Journal of Headache and Pain 2014,15:6

22. Singh A et al. "Central cognitive processing assessed by P300 in migraine, tension-type headache, and cluster headache". Int J Clin Exp Physiol 2015;2:220-3

23. Lifang Huang, Hong juan Dong, Xi Wang, Yan Wang and Zheman Xiao. Duration and frequency of migraines affect cognitive function: evidence from neuropsychological tests and event-related potentials. The Journal of Headache and Pain (2017)18:54

24. Marina Titlic1et al. The Event-related Potential P300 in Patients with Migraine. ACTA INFORM MED. 2015 DEC;23(6):339-42

25. Lipton RB, Stewart WF, Sawyer, J. and Edmeads, J.G. (2001), Clinical Utility of an Instrument Assessing Migraine Disability: The Migraine Disability Assessment (MIDAS) Questionnaire. Headache: The Journal of Head and Face Pain, 41:854-61. doi:10.1111/j.15264610.2001.01156.x 